\title{
Research and application of process reinforcing in welding numerical simulation
}

\author{
Yuanbin $\mathrm{FANG}^{1,2, a}$, Xianfei MENG ${ }^{3, \mathrm{~b}}$, Jinbo $\mathrm{LV}^{3, \mathrm{c}}$, Jun $\mathrm{SHI}^{3, \mathrm{~d}}$ \\ ${ }^{1}$ XCMG, Jiangsu Xuzhou Engineering Machinery Research Institute, Jiangsu Xuzhou 221004, \\ China \\ ${ }^{2}$ State key Laboratory of Intelligent Manufacturing of Advanced Construction Machinery, XCMG \\ Construction Machinery Co., Ltd, Jiangsu Xuzhou 221004, China \\ ${ }^{3}$ XCMG, Xuzhou Heavy Machinery Co., Ltd, Jiangsu Xuzhou 221004, China \\ afybflying@163.com, ${ }^{b}$ meng2001@163.com, calaupo@139.com, ${ }^{\mathrm{d}}$ shijunhfut@163.com
}

\begin{abstract}
Keywords: Process reinforcing; finite element method; equivalent forms; welding deformation Abstract. Taking the turntable of the crane as the object, it simulates the equivalent forms of process reinforcing in the large floor of the turntable. And it obtains welding deformation. By simulating the equivalent forms of process reinforcing, it discusses the influence on welding deformation by rigid fixed, contact, the unidirectional deformation constraint and adhesive contact. It verifies the results by experiments. The results show that they design four groups of the equivalent models, and calculate the welding deformation results of the large floor. When process reinforcing is equivalent to rigid fixed and contact, it predicts the deformation greatly. When process reinforcing is equivalent to the unidirectional deformation constraint and adhesive contact, it predicts deformation relatively small. By adhesive contact, deformation distribution is consistent with measured results, and error is within $6 \%$. It verifies equivalent method is practical in the finite element analysis.
\end{abstract}

\section{Introduction}

Welding deformation is an urgent problem in the field of engineering machinery. In the welding process of complex structure, welding deformation caused by the former procedure will lead to dimension tolerance in the next procedure welding of the parts. Manufacturing departments need to adopt the anti-deformation tooling, welding sequences optimization, welding parameters optimization, and process reinforcing to reduce deformation, even increase the orthopedic procedure [1]. The former three methods need the experience, simulation or experimental study to analyze. Process reinforcing, as a positioning mode effectively, only needs to apply in a larger deformation position, and is widely used in engineering. However, to ensure the robot welding rate and the convenience of manual welding, it is necessary to effectively exert the numbers and the positions of process reinforcing. To avoid a large number of experimental jobs, numerical simulation has become an effective means. It is generally believed that process reinforcing is equivalent to rigid fixed [2].

To better study the influence of process reinforcing on welding quality, many scholars have done many jobs. Based on the finite element method, the reference [3] designed different forms of reinforcing, and the equivalent way applied the axial constraint. It got the rule of welding deformation in the axial direction, radial direction and circumference direction by reinforcing. At the same time, it compared the influence of different kinds of reinforcing on controlling deformation. The reference [4] sums up the law of angular deformation, the experiment has tried to find out the angle deformation by process reinforcing, and obtained the data after increasing the process reinforcing. It has carried on the experiment verification, and controlled the welding angle distortion.

The above literatures show that in the experimental research, it has carried out research work of process reinforcing in the analysis of test and simulation. Although it simulated different forms of process reinforcing, it did not test. In the experimental research, more deformation datas are accumulated by measurement data, and there is no effective deformation prediction by simulation. 
Moreover, the method of applying reinforcing is applied more in the form of stiffened panels with large sections.

Based on the turntable as the object, it is equivalent to the smaller contact area stretching by rigid fixed, contact, the unidirectional deformation constraint and adhesive contact. It establishes finite element model of the turntable. There are compared with experiment results and simulation results of deformation.

\section{Establishment of finite element model}

The turntable is welded by single-pass welding. The material is Q460, and the welding wire uses SLD-60 solid cored wire. The welding method is carbon-dioxide arc welding. The welding voltage is $27-29 \mathrm{~V}$, and the welding current is $280-290 \mathrm{~A}$. The welding speed is $7 \mathrm{~mm} \bullet \mathrm{s}-1$. To avoid the accident caused by experimental operation and other factors, it selects two groups of specimens.

The thermo-physical properties and mechanical parameters change with temperature. The accuracy of the curves decides the accuracy of simulation results. The material properties of Q460 are shown in Fig. 1.

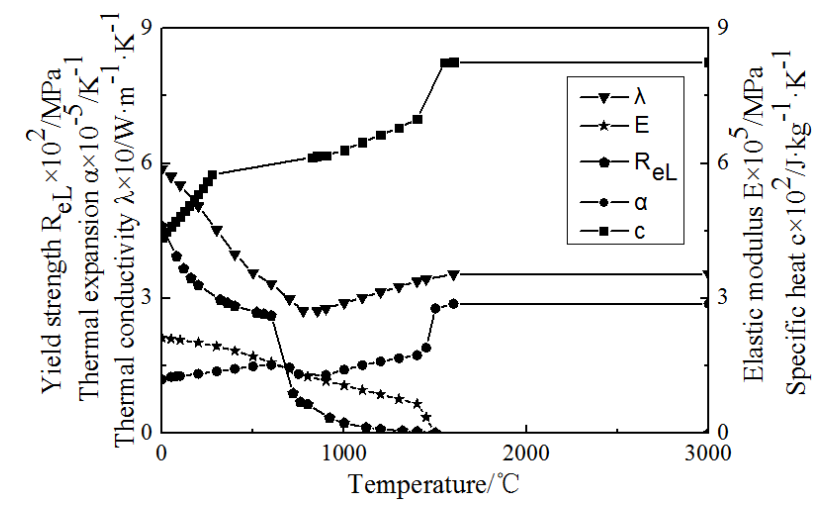

Fig.1 Relationship between thermo-mechanical parameters and temperature of Q460

The thickness of the large floor is $25 \mathrm{~mm}$, and the thickness of the ear plate is $16 \mathrm{~mm}$. The rest is $10 \mathrm{~mm}$. The structure of the turntable is symmetrical, and the half is analyzed. The model is tested by one to one. To verify the influence of process reinforcing on large floor deformation, it measures the distance between point $\mathrm{A}$ and point $\mathrm{B}$. The mesh is hexahedral, and adopts the transition mesh. The maximum size is $30 \mathrm{~mm}$, and the weld seam size is $0.5 \mathrm{~mm}$. To ensure that the mesh size is uniform in the weld seam and heat affected zone, it obtains 376647 grids and 465109 nodes shown in Fig. 2.

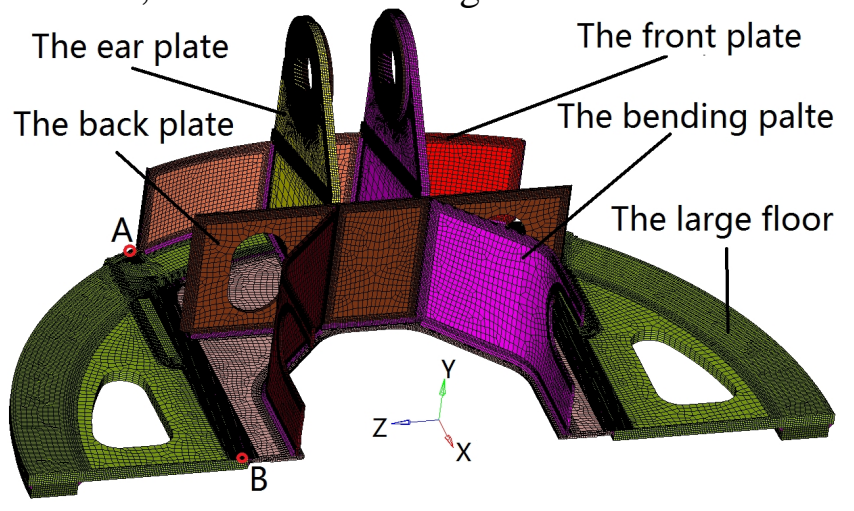

Fig.2 FE model

It selects from the double ellipsoid heat source physical model proposed by Goldak. It adopts steady heat source loading method [5]. The filled grids adopt the preset element life and death element method. There is heat exchange between the surface of the turntable and surrounding environment. And it defines the equivalent boundary conditions of temperature field. The coefficient of heat dissipation is 0.02 , and the ambient temperature is set at a constant temperature of 20 degrees.

In a typical joint, the three-point constraint is applied to prevent the rigid-body displacement. The turntable uses the angle connection form. The deformation of the large floor is small. The constraint 
usually selects the position of big rigid. However, the turntable focuses on the deformation of the large floor. Three-point constraint will is insufficient. The constraint location defines in the part of contact plane. The back position of the large floor corresponding to the ear plate chooses some nodes to limit $\mathrm{XYZ}$ direction. The back position corresponding to another ear plate chooses some nodes to limit YZ direction. The lower position of the symmetrical plane in the large floor chooses some nodes to limit $Y$ direction. Establish the mechanical constraint condition. The contact position between the turntable and the displacement machine is equivalent to the contact between the platform and the turntable.

To simulate the equivalent model of process reinforcing, they adopt four methods, such as, contact, the unidirectional deformation constraint and adhesive contact as shown in Fig. 3.

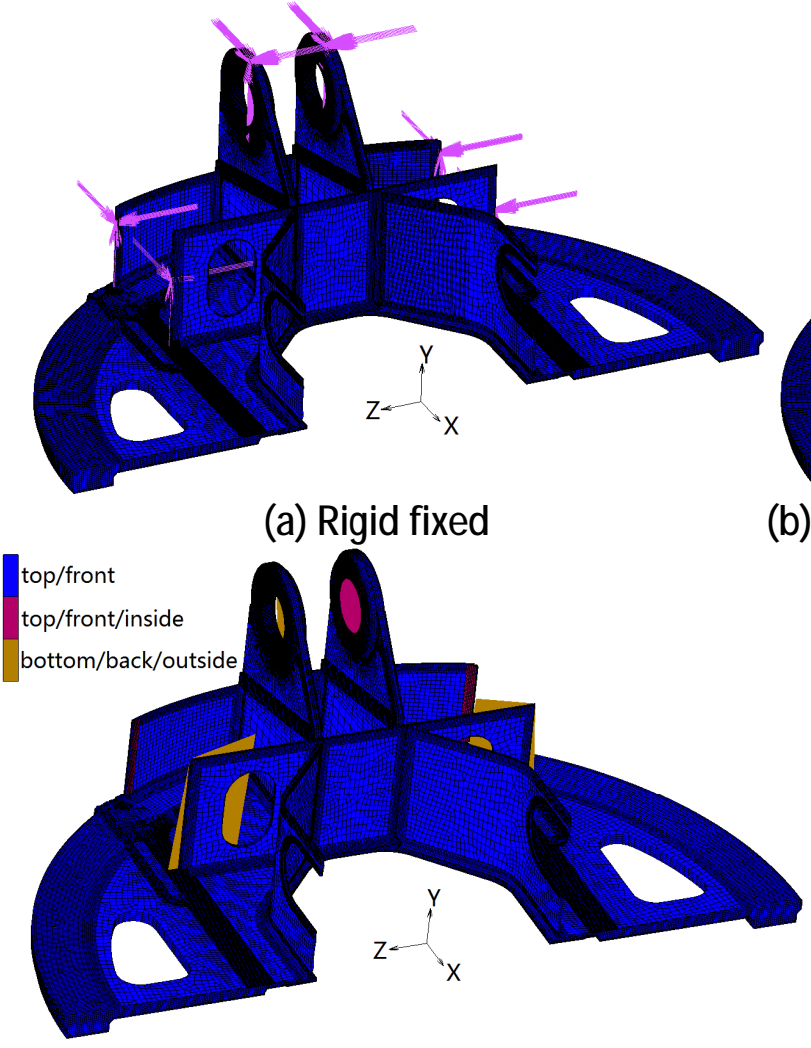

(c) Contact

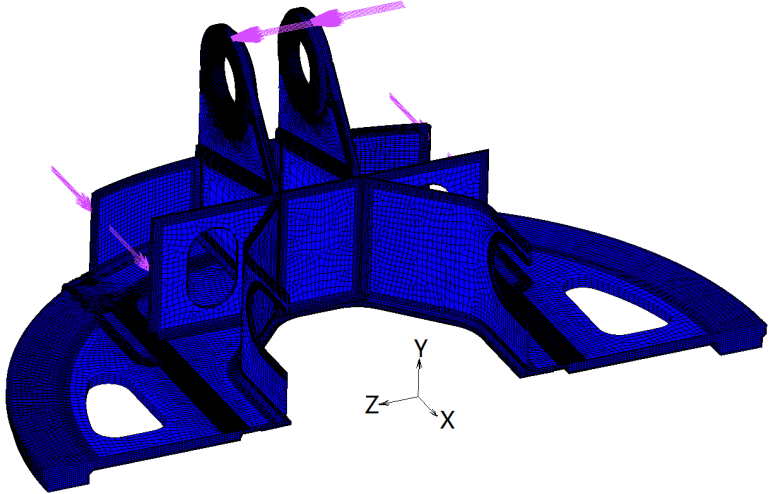

(b) The unidirectional deformation constraint

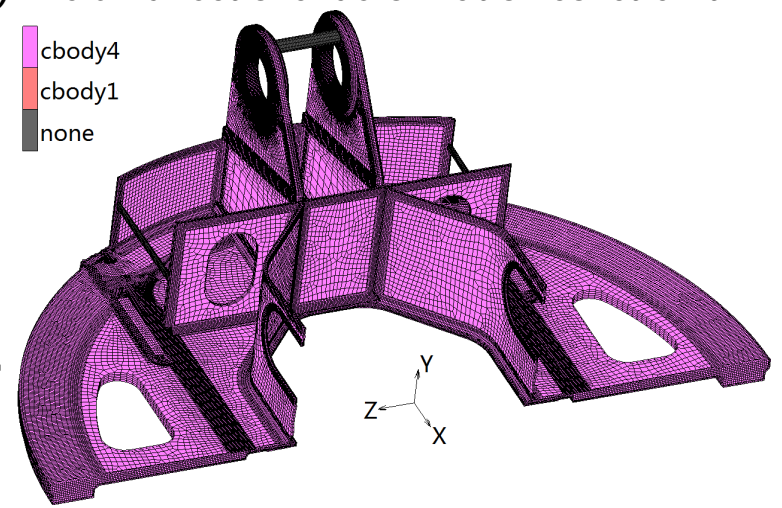

(d) Adhesive contact

Fig.3 Different constraints set of model

The first scheme is that process reinforcing adopts rigid fixed, and the constraint uses the $\mathrm{XYZ}$ displacement constraints. The second scheme is that process reinforcing adopts the unidirectional deformation constraint, and constraints the axial direction of reinforcing. The third scheme is that process reinforcing adopts contact, and the connection position of process reinforcing defines surfaces. The fourth scheme is that it applies adhesive contact. Process reinforcing defines the rigid-body. Different equivalent constraints releases under heat release condition.

\section{Result analysis and discussion}

Influence of different equivalent methods on welding deformation. Through four groups of equivalent model, they calculate the influence of different equivalent models on deformation of the large floor as shown in Fig. 4.

From Fig. 4, in the first and third schemes, when process reinforcing is equivalent to rigid fixed and contact, it predicts the deformation greatly. When process reinforcing is equivalent to the unidirectional deformation constraint and adhesive contact, it predicts deformation relatively small. Rigid fixation and contact make rigid too large in the reinforcing position. The contraction force dues to the front and back plate welding. Welding deformation cannot be released, and the deformation of the large floor makes increases. The constraint effect is greater, and deformation is larger. The restraint will cause a certain moment effect on the large floor deformation, so that the large floor deformation is 
large. Unidirectional displacement constraint and adhesive contact restrict freedom degree weak. The contraction effect along the $\mathrm{Z}$ direction and $\mathrm{X}$ direction is smaller, so that it can effectively simulate the actual condition. The moment influence on the large floor is small, and deformation is small. Process reinforcing is mainly to increase the rigidity. The contact area is small, and the moment of inertia is small.

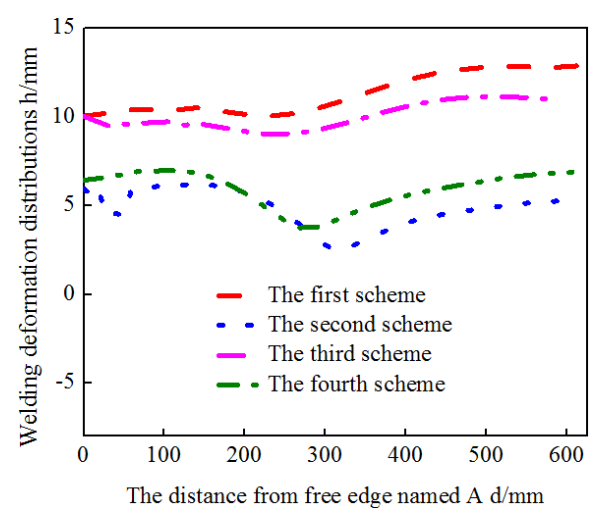

Fig.4 Welding deformation distributions

From the diagram, the deformation of the large floor has a downward trend in the position of $300 \mathrm{~mm}$ from the free edge. This is mainly contraction effect of the bending plate, which makes the large floor deformation along the negative $\mathrm{Y}$ direction.

Test verification. Based on the welding operation guide, it designs the test. It measures the peak value of deformation between point A and B. Many factors influence the turntable deformation, so it uses random sampling method to verify the It calculates average value of the peak difference. It records 2 groups of results. It extracts deformation peak of four schemes as shown in table 1 . The turntable test is verified as shown in Fig. 5.

Table 1 Deformation measurement of big floor

\begin{tabular}{cccc}
\hline The scheme & Maximum value $[\mathrm{mm}]$ & Minimum value $[\mathrm{mm}]$ & difference value $[\mathrm{mm}]$ \\
\hline $\mathrm{A}_{1}$ & 7.2 & 3.5 & 3.7 \\
$\mathrm{~A}_{2}$ & 6.5 & 3.4 & 3.1 \\
1 & 12.9 & 10 & 2.9 \\
2 & 6.19 & 2.55 & 3.64 \\
3 & 11.2 & 9 & 2.2 \\
4 & 6.9 & 3.7 & 3.2 \\
\hline & & &
\end{tabular}

Fig.5 Experimental verification

From table 1, it shows that the flatness average value of the large floor is $3.4 \mathrm{~mm}$. It is proved by experiment that the maximum deformation occurs at both ends of $\mathrm{A}$ and $\mathrm{B}$, and the minimum deformation is near the end of the back plate.

The trend and results of the scheme 4 are more consistent with the results, and the error is less than $6 \%$. The accuracy is mainly for several reasons, such as stable environment temperature, heat dissipation approaching to assuming coefficient, and the stability of welding parameters. There is no complicated boundary condition. The model is not simplified. The grid uniformity is better, and the grid dispersion is higher near the weld seam. 


\section{Conclusions}

Through the simulation of process reinforcing, it designs four groups of equivalent models that are rigid fixed, contact, the unidirectional deformation constraint and adhesive contac. They calculate deformation distributions. Comparing with measurement results, it verifies the equivalent form of process reinforcing. The following conclusions are drawn in the research:

(1) When process reinforcing is equivalent to rigid fixed and contact, they get the similar results and predict the deformation greatly. When process reinforcing is equivalent to the unidirectional deformation constraint and adhesive contact, they get the similar results and predict deformation relatively small.

(2) By adhesive contact, deformation distribution is consistent with test results.

In this study, the calculation accuracy of adhesive contact is high, but the calculation efficiency is reduced. Through the research, it is suggested that it will adopt the unidirectional deformation constraint to simulate process reinforcing, if it predicts the deformation trend. The calculation accuracy and the calculation efficiency can be effectively balanced.

\section{Acknowledgements}

This work was financially supported by the Jiangsu Natural Science Foundation (BK20140229), and National Science and Technology Support Program (2015BAF07B02).

\section{References}

[1] X. T. Tian: Welding Structure (China Machine Press Publications, China 1982).

[2] D. Deng, H. Murakawa, and W. Liang: Journal of Materials Processing Technology, Vol. 203 (2008), p. 252-266.

[3] J. Y. Li, F. L. Sun, Y. Liu, and X. L. Hu: Journal of Harbin University of Science and Technology, Vol. 20, No. 3 (2015), p. 56-60.

[4] X. M. Cao, and X. J. Feng: Metal Working, No. 2 (2015), p. 71-73.

[5] Y. B. Fang, X. M. Zong, H. Q. Zhang, and X. Q. Yin: Electric Welding Machine, Vol. 46, No. 6 (2016), p. 75-79. 